\title{
Image Steganography by Using Multiwavelet Transform
}

\author{
Iman M.G. Alwan* \\ Received 20, December, 2012 \\ Accepted 11, March, 2014
}

\begin{abstract}
:
Steganography is the art of secret communication. Its purpose is to hide the presence of information, using, for example, images as covers. The frequency domain is well suited for embedding in image, since hiding in this frequency domain coefficients is robust to many attacks. This paper proposed hiding a secret image of size equal to quarter of the cover one. Set Partitioning in Hierarchal Trees (SPIHT) codec is used to code the secret image to achieve security. The proposed method applies Discrete Multiwavelet Transform (DMWT) for cover image. The coded bit stream of the secret image is embedded in the high frequency subbands of the transformed cover one. A scaling factors $\alpha$ and $\beta$ in frequency domain control the quality of the stego images. The proposed algorithm is compared with wavelet based algorithm which shows a favorable results in terms of PSNR reaches to $18 \mathrm{~dB}$.
\end{abstract}

Key words: Discrete Multiwavelet Transform (DMWT), image hiding, SPIHT algorithm

\section{Introduction:}

A tremendous work is going on at present to resolve the problems in network security, quality of service control and secure communications through public and private channels. Steganography is branch of information hiding which has found to be useful in fmding the solution to network security. Steganography provides privacy for intelligence, and military personal for those people who are subject to censorship. The purpose of steganography is to send secret data by embedding it into some innocuous cover objects such as digital images or any other medium like audio, video etc. in such a way that it cannot be detected by any third party. To meet it one must fulfill the four basic requirements of steganography: Capacity (the amount of data that can be hidden in the cover-object), Security (degree of detectivity of embedded data by attacker) and Imperceptibility (related to the inability in the detection of hidden information inside a cover by attacker), and Robustness (the amount of modifications that the media can withstand without destroying the message). In almost all steganographic algorithms, data is first encoded with a coding scheme and then the resulted secret data bit are embedded into the cover object using an embedding algorithm. The cover object after embedding is called stego-object. There is likely to be a chance of visibility of embedded data in the cover object if the amounts of change in the cover object while embedding is large enough[1].

To meet the requirement of robustness, we have used transform domain based embedding technique. The transform embedding methods are found to be better than the spatial domain because the transform domains not only have the potential to achieve higher embedding capacity but are also more robust. We can fmd a lot of work on steganography based on different transforms, such as Discrete Cosine

\footnotetext{
*Computer Science Dep., College of Education for Women, Baghdad University
} 
Transform, Fast Fourier Transform, wavelet transform and multiwavelet transform[2].

The transformation can be applied to the entire image or to its subparts. The embedding process is done by modifying some coefficients that are selected according to the type of protection needed. If we want the message to be imperceptible then the high range of frequency spectrum is chosen, but if we want the message to be robust then the low range of frequency spectrum is selected [3].

Contribution of this paper as, the secret image has been lossless compressed by using SPIHT algorithm in order to embed the compressed stream into the cover image, the SPIHT algorithm is very efficient for image compression with high quality reconstructed one. The coded bit stream is embedded in the details subbands of the multiwavelet coefficients of the cover image. Khashandarag and ebrahimian [4] presented a method based on LempelZiv-Welch (LZW) compression and set partitioning in hierarchical trees (SPIHT) codec to obtain a low bit rate and high constructed quality image compression. In the embedding process, an adaptive phase modulation (APM) mechanism and discrete Fourier transform (DFT) were adopted for secret data embedding. Yang $C$. et.al. [5] investigated The magnitude distribution law of DWT coefficients of color image data in Karhunen-Loeve space

Based on their characteristic, a novel ordered-SPIHT for embedded color image coding (OCSPIHT) is proposed in this paper. The set of DWT coefficients of an image in KL space are split into several sets according to their magnitudes. For each specific threshold, only the sets with coefficients equal to or larger than the threshold will be encoded. This can save many bits and improve coding performance especially at low bit rate.

\section{Discrete Multiwavelet Transform}

Historically, the concept of wavelets is originated from the study of time-frequency signal analysis, wave propagation, and sampling theory. The idea of wavelets introduced as a family of functions constructed by using translation and dilation of a mother wavelet for the analysis of nonstationary signals [6]. One generalization of wavelets is multiwavelets [7], which have been around since the early 1990s. Unlike the scalar case, some extra degrees of freedom are allowed, which can be used to construct functions with several desirable properties, combining, for example, orthogonality, symmetry, short support and vanishing moments [8]. All these properties are needed for efficiently processing twodimensional signals, hence multiwavelets are more powerful than wavelet in image processing. In the other hand the application of the multiwavelet

decomposition/reconstruction scheme requires two additional steps respect to the scalar case [6]. In practice multiscaling and wavelet functions often have multiplicity $r=2$. An important example was constructed by Geronimo, Hardin and Massopust which we shall refer to as the GHM system. For the GHM multiscaling functions there are two scaling functions $\varnothing_{1}(\mathrm{t}), \varnothing_{2}(\mathrm{t})$ and the two wavelets $\mathrm{w}_{1}(\mathrm{t}), \mathrm{w}_{2}(\mathrm{t})[9]$.

Before the operation of decomposition is applied to the input data, the preprocessing operation must be done. The aim of preprocessing is to associate the given scalar input signal of length $\mathrm{N}$ to a sequence of length-2 vectors $\{\mathrm{v} 0, \mathrm{k}\}$ in order to start the 
analysis algorithm. Here $\mathrm{N}$ is assumed to be a power of 2 , and so is of even length. After the wavelet reconstruction (synthesis) step a postfilter is applied. Clearly, prefiltering, wavelet transform, inverse transform, and postfiltering should recover the input signal exactly if nothing else has been done. A different type of preprocessing was suggested such as repeated row (oversampling), matrix approximation (critical sampling) and so on [11]. The difference between wavelet and multiwavelet is shown in Fig. 1.
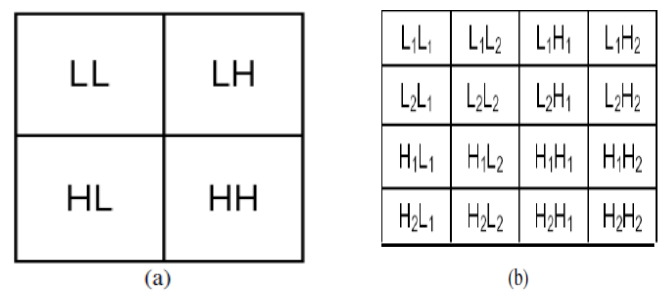

(b)

Fig. 1 Image sub bands after a single-level decomposition, for (a) scalar wavelet, (b) multiwavelet

Analysis and synthesis of input signal using multiwavelet transform filters are shown in Fig. 2.

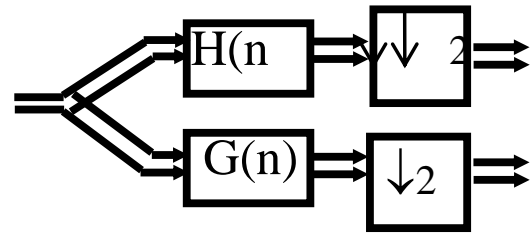

Analysis

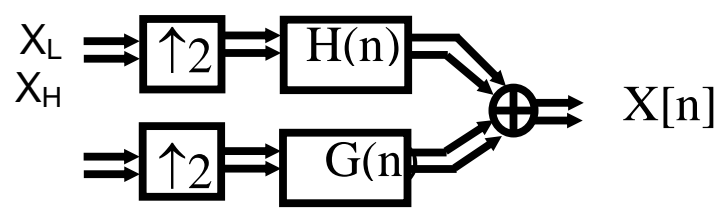

Synthesis

Fig. 2 Analysis and synthesis of input signal using multiwavelet transform

\section{The Set Partitioning In Hierarchical}

\section{Trees Algorithm:}

It was invented by Said and Pearlman in 1996 [12]. SPIHT was designed for optimal progressive transmission, as well as for compression. One of the important features of SPIHT is that at any point during the decoding of an image, the quality of the displayed image is the best that can be achieved for the number of bits input by the decoder up to that moment. Another important SPIHT features is its use of embedded coding. It is a wavelet-based compression method. After performing the wavelet transform, SPIHT is used to encode the wavelet coefficients. It exploits the organization of wavelet coefficients into the spatial orientation trees. This structure is defined as a special relationships between the wavelet coefficients in the different levels of the subband pyramid, where subbands in each level of the pyramid exhibits spatial similarity. Any special features, such as a straight edge or a uniform region, are visible in all the levels at the same location. Fig.(3) shows the relationship of the type A and type $\mathrm{B}$, where type $\mathrm{B}$ is a sub-tree of type A. Fig.(4) shows the coefficients of $\mathrm{A}(1,1)$ and $\mathrm{B}(1,1)$, A $(1,1)$ is a 4 pixels block expanded from the pixel $(1,1)$ by SPIHT, and the other sixteen pixels block expanded from the pixel $(1,1)$ by SPIHT is called $\mathrm{B}(1,1)$. 


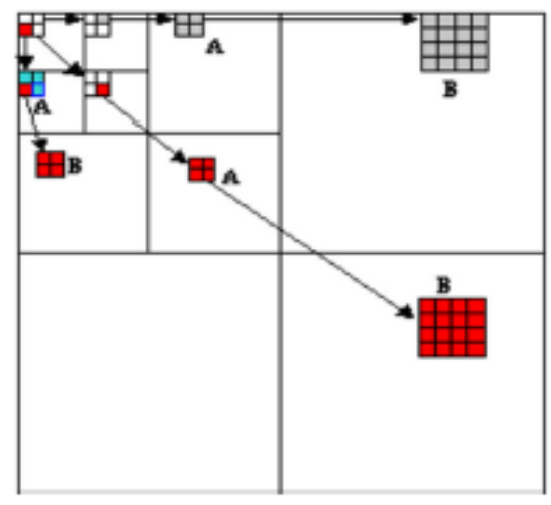

Fig. 3. The relationship of the type $A$ pixels and type $B$, type $B$ is sub-tree of type $A$

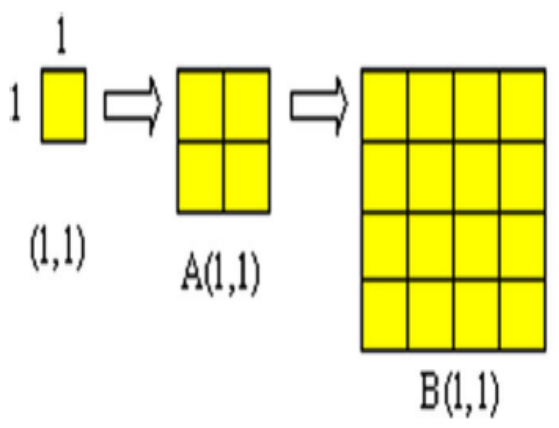

Fig. 4. Corresponding to the pixel $(1,1)$, the four called $A(1,1)$ and the sixteen pixels is called $B(1,1)$.

The algorithm of SPIHT algorithm is specified as follows [12] :

$O(i, j)$ :set of coordinates of all offspring of node $(i, j)$.

$D(i, j)$ : set of coordinates of all descendants of node $(i, j)$.

$H$ : set of coordinates of all spatial orientation tree roots (nodes in the highest pyramid level).

LIS: list of the insignificant sets.

LIP: list of the insignificant pixels.

LSP: list of the significant pixels.

$L(i, j): D(i, j)-O(i, j)$.

A LIS entry is of type A if it represents $D(i, j)$,

represents $L(i, j)$.

type $B$ if it

SPIHT uses the function
$S_{n}(T)=\left\{\begin{array}{cc}1 & \max _{(i, j) \in T}\{|c(i, j)|\} \geq 2^{n} \\ 0 & \text { otherwise }\end{array}\right.$

to indicate the significance of a set of coordinates $T$. The steps of the algorithm are as follows:

(1) Initialization:

Output $n=\left\lfloor\log _{2}\left(\max _{(i, j)}\left\{\left|c_{i, j}\right|\right\}\right)\right\rfloor$

Set the LSP as an empty list;

Add the coordinates $(i, j) \in H$ to the list LIP, and only those with descendants also to the $L I S$, as type A entries.

(2) Sorting pass:

(2.1) for each entry $(i, j)$ in the $L I P$ do;

(2.1.1) transmit $S_{n}(i, j)$

(2.1.2) if $S_{n}(i, j)=1$ then move (i, to the LSP and transmit the sign of $\boldsymbol{C}_{i, j}$;

(2.2) for each entry $(i, j)$ in the LIS do: (2.2.1) if the entry is of type A, then

- Transmit $S_{n}(D(i, j))$

- If $S_{n}(D(i, j))=1$ then

* For each $(k, l) \in O(i, j)$ do:

○ Output $S_{n}(k, l)$

$\circ$ If $S_{n}(k, l)=1$

then add $(k, l)$ to the $L S P$ and output the sign of

$c_{k, l}$

○ If $S_{n}(k, l)=0$

then add $(k, l)$ to the end of the $L I P$;

* If $L(I, j) \neq 0$ then move $(i, j)$ to the end of the $L I S$, as an entry of type B, and go to step (2.2.2);

Otherwise, remove entry $(i, j)$ from the $L I S$; 
(2.2.2) if the entry is of type B, then

- Transmit $S_{n}(L(i, j))$

- If $S_{n}(L(i, j))=1$ then * Add each $(k, l) \in O(i, j)$ to the end of the LIS as an entry of type

A;
LIS $\quad$ Remove $(i, j)$ from the

(3) Refinement pass For each entry $(i, j)$ in the $L S P$, except those included in the last sorting pass, output the $n$th most significant bit of

$\left|c_{i, j}\right|$;

(4) Quantization-step update; decrement $n$ by 1 and go to step (2).

\section{4- The Proposed Algorithm}

A steganography scheme must be extremely secure, and does not reduce the visual quality of the cover image when the secret image is concealed. The proposed algorithm depends on coding the gray scale secret image, which has the (1/4) dimensions as the gray scale cover one, lossless by applying SPIHT algorithm in order to achieve high security for embedding in cover image. The reasons behind using SPIHT algorithm are the good imagequality, high peak signal to noise ratio, simple quantization algorithm, and fast coding/decoding (nearly symmetric) [12]. For embedding a bit stream is transformed into a sequence $w(1) \ldots w(L)$ by replacing the 0 by -1 , where $L$ is the length of the bit stream and $w(k) \in\{-1,1\}$ $(\mathrm{k}=1, \ldots, \mathrm{L})$. This sequence is used as the watermark. The one level wavelet transform is applied for the cover image and the bit stream of the secret image is embedded in the detail subbands of the cover image. It is assumed that the cover image is available for secret image recovery.

\section{Algorithm 1: Secret image embedding}

Input: secret image, Cover image, $\alpha, \beta$ : Scaling factors.

Output: Stego-image.

Step1: Applydiscrete multi wavelet transform for the cover image to obtain the subbands ( $\mathrm{LiLj}, \mathrm{LiHj}, \mathrm{HiLj}, \mathrm{HiHj}$ ) $(\mathrm{i}, \mathrm{j}=1,2)$

Step2: Compress the secret image loosless by applying SPIHT encoding algorithm to get the desired bit stream for embedding.

Step3: transformed the bit stream into a sequence $w(1) \ldots w(L)$ by replacing the 0 by -1 , where $\mathrm{L}$ is the length of the bit stream and $w(k) \in \square\{-1,1\}(\mathrm{k}=1, \ldots, \mathrm{L})$.

Step4: Embed the binary sequence in $\mathrm{LiHj}, \mathrm{HiLj}, \mathrm{HiHj}$ subbands of the cover image according to the formula.

$\mathrm{S}=\alpha^{*} \mathrm{C}+\beta^{*} w$

Where $\mathrm{C}$ : is the multiwavelet of the cover image.

embedded.

$$
w \text { : is the bit stream to be }
$$

Step6: End.

\section{Algorithm 2: Secret image extraction}

It is assumed that the original image, value of $\alpha$, and $\beta$ are known for extraction. Input: Stego-image.

Output: Secret image.

Step1: Apply DMWT for the stego image, to get the subbands LiLj,LiHj,HiLj,HiHj

f.or $(i, j=1,2)$

Step2:Extract the embedded sequence from the subbands $\mathrm{LiHj}, \mathrm{HiLj}, \mathrm{HiHj}$, by applying the equation

$$
w=(\alpha * \mathrm{C}-\mathrm{S}) / \beta
$$

Step3: Convert the extracted sequence into 0,1 sequence .

Step4: Apply the SPIHT decoding algorithm to get the secret image.

Step5: End 


\section{5- The Experimental Results}

The proposed algorithm is implemented and tested on several standard images. The cover images are of size 256x256 (gray images). The secret images are of size $128 \times 128$ (gray images). First the secret image is coded by SPIHT encoder to a rate of 8 bits per pixel (lossless compression) to achieve a maximum bit rate for embedding in the high frequency bands of the cover image. Fig.(5) shows at the first row the secret images. The second row is for the cover images.

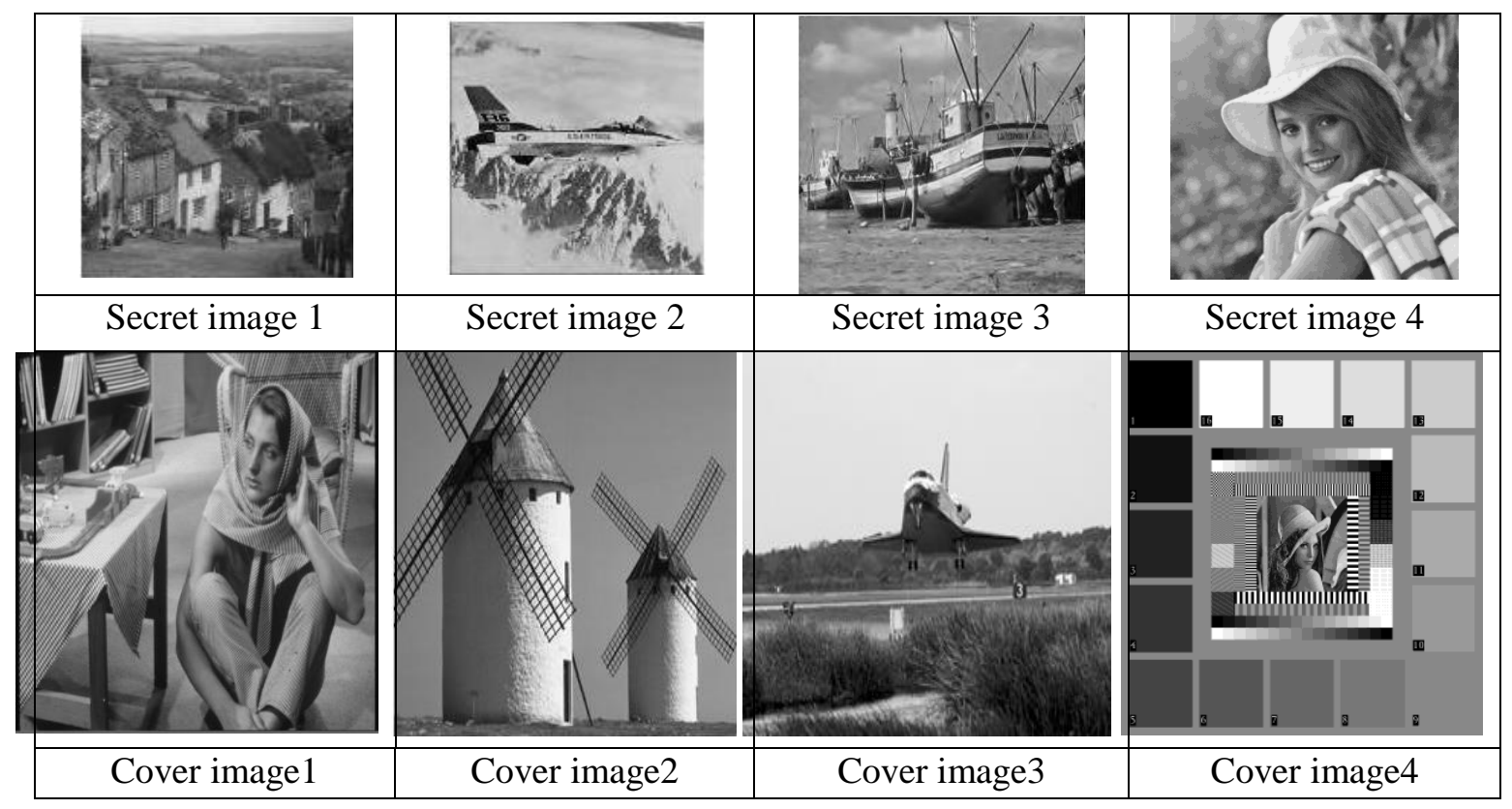

Fig.(5) The test secret and cover images

After the execution of the embedding process, the result is the stego images. For extracting of the secret image, the extracting algorithm is executed. Peak Signal to Noise Ratio (PSNR) measure and Correlation measure [13] are used to evaluate the quality of stego-image, with respect to the original cover image. When the stego-image is perceptually similar to the original cover-image, the correlation equals one. These metrics are defined as follows:

$$
\begin{aligned}
& P S N R=10 \log _{10}\left(\frac{255^{2}}{M S E}\right) \ldots \ldots . . \\
& M S E=\left(\frac{1}{N}\right)^{2} \sum \sum\left(x_{i j}-x_{i j}\right)^{2}
\end{aligned}
$$

Where $x_{i j}$ 's denote the original pixel values, $x_{i j}^{\prime} \mathrm{s}$ denote the modified pixel values, nd $\mathrm{N}$ is the dimension of the image.

$$
\text { Corr. }=\frac{\sum_{i=1}^{M} \sum_{j=1}^{N}(X(i, j)-\bar{X})(Y(i, j)-\bar{Y})}{\sqrt{\left[\sum_{i=1}^{M} \sum_{j=1}^{N}\left(X(i, j)-\overline{X)^{2}}\right]\right.}\left[\sum_{i=1}^{M} \sum_{j=1}^{N}\left(Y(i, j)-\overline{Y)^{2}}\right]\right.}
$$

where $\mathrm{M}, \mathrm{N}$ :height and width of the two images

$X(i, j):$ the original image.

$Y(i, j):$ The modified image.

$\bar{X}$ and $\bar{Y}$ : Mean of original and modified images, respectively.

Fig.(6) shows the stego images and the extracted secret images of the proposed algorithm with the PSNR for each one of them. 


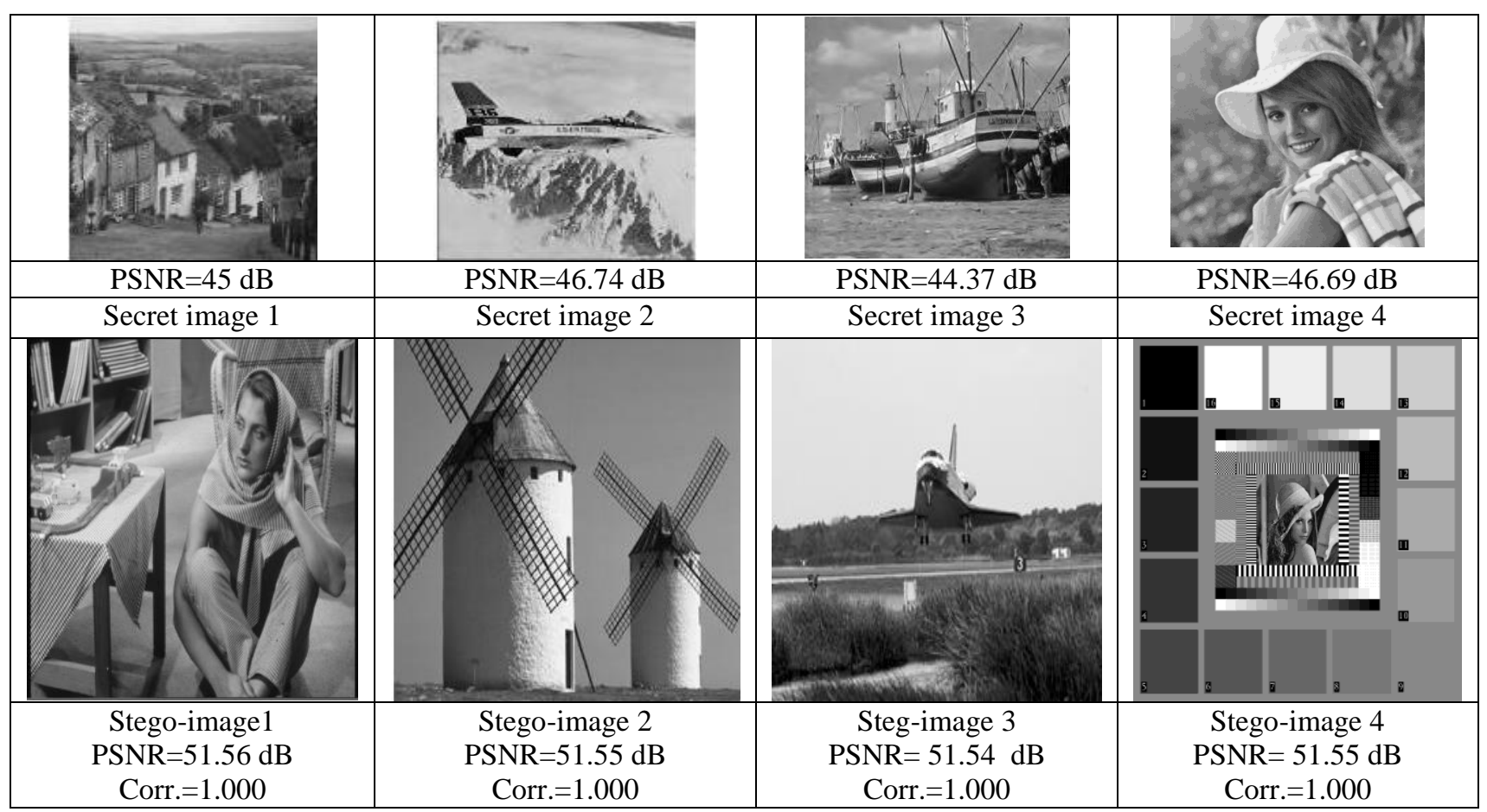

Fig (6) The PSNR for reconstructed secret images and PSNR and Correlation for stego-images

The proposed algorithm is also compared with wavelet based steganography system which shows immense improvement in the PSNR of the stego images for DMWT based system compared with DWT one. The average improvement of PSNR is approximately (18dB). Table(1) shows the result of PSNR for stego and reconstructed secret images for both DMWT and DWT systems for test images used in this paper.

Table(1) PSNR of stego and reconstructed secret images using DMWT and DWT.

\begin{tabular}{|c|c|c|c|c|}
\hline Test images & $\begin{array}{c}\text { PSNR of } \\
\text { Stego-image } \\
\text { (DMWT) }\end{array}$ & $\begin{array}{c}\text { PSNR of } \\
\text { reconstructed } \\
\text { Secretimage(DMWT) }\end{array}$ & $\begin{array}{c}\text { PSNR of steg } \\
\text { (DWT) }\end{array}$ & $\begin{array}{c}\text { PSNR of } \\
\text { reconstructed } \\
\text { secret image } \\
\text { (DWT) }\end{array}$ \\
\hline Number 1 & $51.56 \mathrm{~dB}$ & $45 \mathrm{~dB}$ & $34.02 \mathrm{~dB}$ & $45 \mathrm{~dB}$ \\
\hline Number 2 & $51.55 \mathrm{~dB}$ & $46.74 \mathrm{~dB}$ & $33.33 \mathrm{~dB}$ & $46.74 \mathrm{~dB}$ \\
\hline Number 3 & $51.54 \mathrm{~dB}$ & $44.37 \mathrm{~dB}$ & $35.45 \mathrm{~dB}$ & $44.37 \mathrm{~dB}$ \\
\hline Number 4 & $51.55 \mathrm{~dB}$ & $46.69 \mathrm{~dB}$ & $30.24 \mathrm{~dB}$ & $46.69 \mathrm{~dB}$ \\
\hline
\end{tabular}

\section{6- Conclusion}

In the proposed algorithm, a scheme of embedding image data is presented. It exploits the characteristics of the SPIHT algorithm in coding and compressing the secret image losslessly in order to obtain a coded compressed bit stream to reduce the total number of bits to be hided as well as achieve higher security . This algorithm depends on using discrete multiwavelet transform for cover image. the coded secret data is embedded into High frequency subbands to prevent visual artifacts, leaving the low frequency subbands 
unchanged. This algorithm is compared with wavelet transform based system. The results show superior improvements of PSNR.

The proposed algorithm is more secure because the restriction of the embedding algorithm (i.e., the use of SPIHT coding algorithm, the use of multiwavelet transform and the value of the scaling factors).

\section{References:}

[1] S.K.Mutt S.K. and Kuma S.," Secure Image Steganography based on Slantlet Transform" International Conference on Methods and Models in Computer Science, 2009

[2] Cheddad, A. , Condell, J., Curran, K. and McKevitt P., "Digital image steganography: Survey and analysis of current method" Signal Processing vol. 90, pp. 727-752, 2010.

[3] Tolba M. F., Ghonemy M. A., Taha I. A., Khalifa A. S., "Using integer wavelet transforms in colored image-steganography", IJICIS, Vol. 4, No. 2, July 2004.

[4] Khashandarag A., and ebrahimian N., "A New Method For Color Image Steganography Using SPIHT and DFT, Sending With JPEG Format", International Conference on Computer Technology and Development,IEEE Computer Society,pp. 581-586, 2009.

[5] Yang, C.L. , PO L.M., Cheung C.H., and Cheung K.W.," A novel ordered-SPIHT for embedded color image coding", IEEE International Conference on Neural Networks \& Signal Processing, Vol. 2, PP. 10871090, 2003.

[6] Agreste S., Vocaturo A., "Wavelet and multichannel wavelet based watermarking algorithms for digital color images", Communications to SIMAI congress, ISSN 1827-9015, Vol. 32009.

[7] Bacchelli S., Cotronei .M, and Sauer T., "Multifilters with and without prefilters", BIT 42, 2:231-261, 1998.

[8] Strela V., "Multiwavelets: Theory and Applications", PhD thesis, Massachusetts Institute of Technology, 1996.

[9] AL-Jauhar W. A., AL-Dargazli M. E., "Thresholding Methods For Image Denoising Using Discrete Multiwavelet Transform", Engineering And Technology Journal, Vol. 41, 2004.

[10] Strela V. and Walden A., "Orthogonal and biorthogonal multiwavelets for signal denoising and image compression", Dep. Of Math., Dartmouth College, 6188 Bradley Hall, Hanover, NH 03755, U.S.A., 1999.

[11] Xia X.G., Geronimo J., Hardin D., and Suter B., "On discrete multiwavelet transforms", IEEE Trans. on Signal Processing, 44, 1996.

[12] Said, A. and Pearlman, W.A. " A New Fast and Efficient Image Codec Based on Set Partitioning In Hierarchical Trees", IEEE Trans. Circuits \& Systems for Video Technology, Vol.6, PP. 243-250, June 1996.

[13] Almohammad,A. Hierons, R. M., and Ghinea, G. "High Capacity Steganographic Method Based Upon JPEG," presented at The Third International Conference on Availability, Reliability, and Security, ARES08, pp. 544-549, Barcelona, Spain, 4-7 March, 2008. 
إخفاء الصورة باستعمال تحويلة متعددة المويجات

ايمان محد جعفر*

| - (كلية التربية للبنات، قسم الحاسبات، جامعة بغداد.

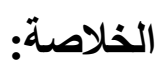

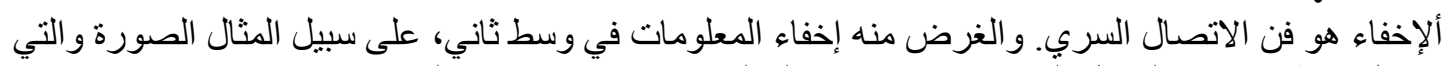

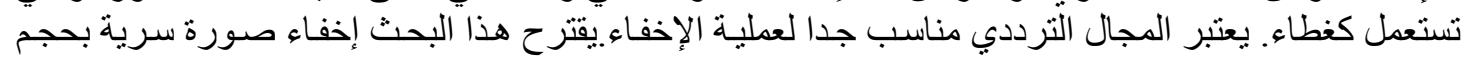

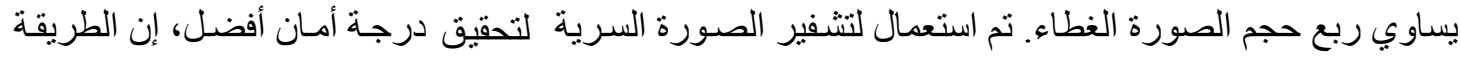

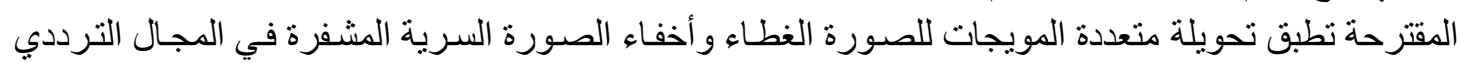
العالي للصورة الغطاء. إن المعاملين ه و م في المجال الترددي يسيطران على جودة الصورة الناتجة. 